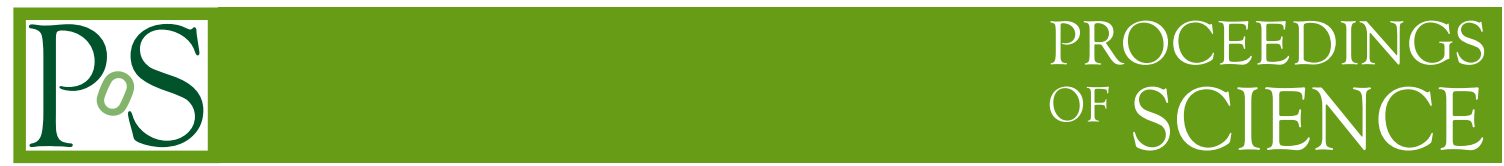

\title{
Noncommutative Baby Skyrmions
}

\section{Theodora loannidou*t}

Department of Mathematics, Physics and Computational Sciences, Faculty of Engineering, Aristotle University of Thessaloniki, Thessaloniki 54124, Greece

E-mail: ti3eauth.gr

\section{Olaf Lechtenfeld}

Institut für Theoretische Physik, Leibniz Universität Hannover Appelstraße 2, 30167 Hannover, Germany

E-mail: lechtenf@itp.uni-hannover.de

A class of exact analytic noncommutative baby Skyrmions is obtained. The corresponding configurations have singular commutative limit but are stable against scaling due to the noncommutativity. We compute their energies, investigate their stability and determine the asymptotic two-Skyrmion interaction.

Corfu Summer Institute on Elementary Particles and Physics - Workshop on Non Commutative Field Theory and Gravity,

September 8-12, 2010

Corfu Greece

\footnotetext{
* Speaker.

${ }^{\dagger}$ The talk is based on our work presented in the paper [1].
} 


\section{Introduction}

In the $\mathbb{C} P^{1}$ baby Skyrme model, the target manifold $S^{2}$ is parametrized by a three-dimensional isovector scalar $\phi$ subject to the constraint $|\phi|^{2}=1$. Its Lagrangian density is of the form

$$
\mathscr{L}=\frac{1}{2} \partial_{\mu} \phi \partial^{\mu} \phi-\frac{\kappa^{2}}{4}\left(\partial_{\mu} \phi \times \partial_{\nu} \phi\right)\left(\partial^{\mu} \phi \times \partial^{v} \phi\right)-V(\phi),
$$

where the field $\phi$ is a map from the three-dimensional Minkowski space $\mathbb{R}^{1,2}$ with the metric $\left(\eta_{\mu v}\right)=\operatorname{diag}(+1,-1,-1)$ to the two-sphere $S^{2}$ of unit radius. The first term in (1.1) is the familiar $\mathbb{C} P^{1}$ sigma model, the second term is the two-dimensional analogue of the Skyrme term and carries a coupling $\kappa$ of the dimension of length, and the last term is the potential, for which different proposals have been made. Finiteness of the energy requires the field to approach a zero of the potential (the 'vacuum' $n$ ) at spatial infinity, allowing one to compactify the static base space $\mathbb{R}^{2}$ to $S^{2}$ and to consider $\phi$ as a map $S^{2} \rightarrow S^{2}$. This gives rise to the homotopy invariant

$$
\operatorname{deg}[\phi]=\frac{1}{4 \pi} \int \mathrm{d} x \mathrm{~d} y \phi \cdot\left(\partial_{x} \phi \times \partial_{y} \phi\right) \in \mathbb{Z},
$$

also known as the topological charge or the Skyrmion number, which is conserved.

The baby Skyrme model is a useful laboratory for studying soliton physics. It is the $2+1$ dimensional analog of a model which describes the low-energy chiral dynamics of Quantum Chromodynamics [2], the usual Skyrme model [3]. This model has direct applications in condensed matter physics [4], where baby Skyrmions give an effective description in quantum Hall systems. In such systems, the dynamics are governed by the spin stiffness term, the Coulomb interaction and the Zeeman interaction. In particular, its kinetic energy corresponds to the spin stiffness term, and the potential (or mass) term corresponds to the Zeeman interaction, the correspondence being exact for the static sector. The Skyrme term is analogous to the Coulomb term. All terms are needed to prevent the collapse of topological configurations which yield to Skyrmion solutions.

In this situation, a noncommutative deformation (for reviews see [5]) may serve as a substitute for the potential term (or Zeeman interaction), because it introduces a new length scale into the theory, which also stabilizes solitons against collapse or spreading. We expect this to give rise to a new class of baby Skyrmions. Indeed, it is known that Moyal-deformed field theories have a much richer soliton spectrum than their commutative counterparts (see, e.g., $[6,7]$ and references therein).

In what follows, based on our work in [1] we present a noncommutative baby Skyrme model, without potential term, for group- or Grassmannian-valued targets, and explicitly obtain a class of exact analytic solitonic solutions, which have no analogues in the commutative theory. This surprising feat succeeds because certain BPS configurations of the Moyal-deformed ordinary sigma model extremize the Skyrme part of the energy as well. We compute their static energy, discuss their stability and evaluate the two-Skyrmion interaction potential at large distances.

\section{The baby Skyrme model}

The $\mathbb{C} P^{1}$ sigma model is the paradigm of a Grassmannian sigma model. A general group-valued or Grassmannian-valued baby Skyrme model then features fields

$$
g: \mathbb{R}^{1,2} \rightarrow \mathrm{U}(n) \text { or } \operatorname{Gr}(n, k) \quad \text { via } \quad\left(x^{\mu}\right) \equiv\left(t, x^{i}\right) \equiv(t, x, y) \mapsto g(t, x, y),
$$


which enter as variables in the action (without potential term)

$$
S=\int \mathrm{d}^{1+2} x\left\{\frac{1}{2} \eta^{\mu v} \partial_{\mu} g^{\dagger} \partial_{\nu} g+\frac{\kappa^{2}}{4}\left[g^{\dagger} \partial_{\mu} g, g^{\dagger} \partial_{v} g\right]\left[g^{\dagger} \partial^{\mu} g, g^{\dagger} \partial^{v} g\right]\right\} .
$$

Classical solutions are obtained by solving the equation of motion

$$
\partial^{\mu} j_{\mu}=0 \quad \text { for } \quad j_{\mu}=g^{\dagger} \partial_{\mu} g+\kappa^{2}\left[g^{\dagger} \partial^{v} g,\left[g^{\dagger} \partial_{\mu} g, g^{\dagger} \partial_{\nu} g\right]\right] .
$$

Let us concentrate on static solutions, $\partial_{t} g \equiv 0$, which are found by extremizing the energy

$$
E=\int \mathrm{d}^{2} x\left\{\frac{1}{2} \partial_{i} g^{\dagger} \partial_{i} g-\frac{\kappa^{2}}{4}\left[g^{\dagger} \partial_{i} g, g^{\dagger} \partial_{j} g\right]\left[g^{\dagger} \partial_{i} g, g^{\dagger} \partial_{j} g\right]\right\} .
$$

For Grassmannian models, this simplifies since $\operatorname{Gr}(n, k)$ is embedded in $\mathrm{U}(n)$ via the constraint

$$
g^{2}=\mathbb{1}_{n} \quad \Leftrightarrow \quad g^{\dagger}=g \quad \Leftrightarrow \quad g=\mathbb{1}_{n}-2 P \quad \text { with } \quad P^{\dagger}=P=P^{2},
$$

and so their energy becomes

$$
E_{\mathrm{Gr}}=\int \mathrm{d}^{2} x\left\{2 P_{i} P_{i}-4 \kappa^{2}\left[P_{i}, P_{j}\right]\left[P_{i}, P_{j}\right]\right\},
$$

where the standard notation $\partial_{i} P=P_{i}, \partial_{i} \partial_{j} P=P_{i j}$ was introduced. We are looking for extrema of the energy (2.4) which are located inside some Grassmannian. Putting $\delta E=0$ and employing (2.5), in particular $g^{\dagger} \partial g=-2[\partial P, P]$ and $\partial_{i}\left(g^{\dagger} \partial_{i} g\right)=-2\left[P_{i i}, P\right]$, one gets

$$
\begin{gathered}
{\left[P_{i i}, P\right]+4 \kappa^{2} F[P]=0 \quad \text { with }} \\
F[P]=2 P_{i j}\left[P_{i}, P\right] P_{j}-\partial_{i}\left(P_{j} P_{j}\right)\left[P_{i}, P\right]+P_{j}\left[P_{i i}, P\right] P_{j}-P_{j} P_{j}\left[P_{i i}, P\right]-\text { h.c. } .
\end{gathered}
$$

Solutions to (2.7) extremize the energy (2.6) of the $\operatorname{Gr}(n, k)$ model as well as (stronger) the energy (2.4) of the $\mathrm{U}(n)$ model. From now on we pass to complex coordinates $z=x+\mathrm{i} y$ and $\bar{z}=x-\mathrm{i} y$. At $\kappa=0$ we connect with the ordinary sigma model. Grassmannian-valued extrema of its energy are provided by the well known BPS projectors, defined through

$$
0=\left(\mathbb{1}_{n}-P\right) P_{\bar{z}}=P_{\bar{z}} P \quad \Longleftrightarrow \quad 0=P_{z}\left(\mathbb{1}_{n}-P\right)=P P_{z} .
$$

These relations (together with $P^{2}=P$ ) imply various useful identities, such as $\left[P_{z}, P_{\bar{z}}\right]=P_{z \bar{z}}$ and

$$
0=\left(\mathbb{1}_{n}-P\right) P_{\bar{z} \bar{z}}=P_{\bar{z} \bar{z}} P=P_{z z}\left(\mathbb{1}_{n}-P\right)=P P_{z \bar{z}}=P_{z} P_{z}=P_{\bar{z}} P_{\bar{z}}=\left[P_{z \bar{z}}, P\right] .
$$

We now turn $\kappa$ back on and compute the failure of the BPS projectors to extremize the baby Skyrme energy:

$$
\frac{1}{8} F[P \text { subject to (2.9) }]=K_{z} P_{\bar{z}}-K_{\bar{z}} P_{z}-P_{z} K_{\bar{z}}+P_{\bar{z}} K_{z}=P_{\bar{z}} P_{z z} P_{\bar{z}}-P_{z} P_{\bar{z} \bar{z}} P_{z}
$$

with the definition $K \equiv \frac{1}{4} P_{i} P_{i}=\frac{1}{2}\left(P_{z} P_{\bar{z}}+P_{\bar{z}} P_{z}\right)$.

To get a feeling, we evaluate this expression in the $\mathbb{C} P^{1}$ model for the (rank-one) BPS projectors, which are based on holomorphic functions $f$,

$$
\frac{1}{8} F=\frac{1}{(1+f \bar{f})^{4}}\left(\begin{array}{cc}
\bar{f} f^{\prime 2} \bar{f}^{\prime \prime}-f \bar{f}^{\prime 2} f^{\prime \prime} & \bar{f}^{2} f^{\prime 2} \bar{f}^{\prime \prime}+\bar{f}^{\prime 2} f^{\prime \prime}-2 \bar{f} f^{\prime 2} \bar{f}^{\prime 2} \\
-f^{2} \bar{f}^{\prime 2} f^{\prime \prime}-f^{\prime 2} \bar{f}^{\prime \prime}+2 f f^{\prime 2} \bar{f}^{\prime 2} & f \bar{f}^{\prime 2} f^{\prime \prime}-\bar{f} f^{\prime 2} \bar{f}^{\prime \prime}
\end{array}\right) .
$$

This vanishes only for constant $f$. We conclude that the sigma-model BPS solitons never obey the baby Skyrme equation of motion. 


\section{Moyal deformation}

A Moyal deformation of Euclidean $\mathbb{R}^{2}$ with coordinates $(x, y)$ is achieved by replacing the ordinary pointwise product of smooth functions on it with the noncommutative but associative Moyal star product. The latter is characterized by a constant positive real parameter $\theta$ which prominently appears in the star commutation relation between the coordinates,

$$
x \star y-y \star x \equiv[x, y]_{\star}=\mathrm{i} \theta \quad \Longrightarrow \quad[z, \bar{z}]_{\star}=2 \theta .
$$

It is convenient to work with the dimensionless coordinates

$$
a=\frac{z}{\sqrt{2 \theta}} \quad \text { and } \quad a^{\dagger}=\frac{\bar{z}}{\sqrt{2 \theta}} \quad \Longrightarrow \quad\left[a, a^{\dagger}\right]_{\star}=1 .
$$

For a concise treatment of the Moyal star product see [5].

A different realization of this Heisenberg algebra promotes the coordinates (and thus all their functions) to noncommuting operators acting on an auxiliary Fock space $\mathscr{H}$ but keeps the ordinary operator product. The Fock space is a Hilbert space with orthonormal basis states

$$
\begin{gathered}
|m\rangle=\frac{1}{\sqrt{m !}}\left(a^{\dagger}\right)^{m}|0\rangle \quad \text { for } \quad m \in \mathbb{N}_{0} \quad \text { and } \quad a|0\rangle=0, \\
a|m\rangle=\sqrt{m}|m-1\rangle, \quad a^{\dagger}|m\rangle=\sqrt{m+1}|m+1\rangle, \quad N|m\rangle:=a^{\dagger} a|m\rangle=m|m\rangle,
\end{gathered}
$$

therewith characterizing $a$ and $a^{\dagger}$ as standard annihilation and creation operators. The star-product and operator formulations are tightly connected through the Moyal-Weyl map: Coordinate derivatives correspond to commutators with coordinate operators,

$$
\sqrt{2 \theta} \partial_{z} \leftrightarrow-\operatorname{ad}\left(a^{\dagger}\right) \quad, \quad \sqrt{2 \theta} \partial_{\bar{z}} \leftrightarrow \operatorname{ad}(a)
$$

and the integral over the noncommutative plane reads

$$
\int \mathrm{d}^{2} x f_{\star}(x)=2 \pi \theta \operatorname{Tr}_{\mathscr{H}} f_{\text {op }},
$$

where the function $f_{\star}$ corresponds to the operator $f_{\text {op }}$ via the Moyal-Weyl map and the trace is over the Fock space $\mathscr{H}$. We shall work with the operator formalism but refrain from introducing special notation indicating operators, so all objects are operator-valued if not said otherwise. The time coordinate $t$ of the full baby Skyrme model remains commutative. Hence, we trade the spatial dependence of our fields with operator valuedness (in $\mathscr{H}$ ), and thus work with maps from the time interval into an enlarged target space, namely $\mathrm{U}\left(\mathbb{C}^{n} \otimes \mathscr{H}\right)=\mathrm{U}(\mathscr{H} \oplus \ldots \oplus \mathscr{H})$ or some Grassmannian subspace thereof.

Since the noncommutative target space is much bigger than the original one, new possibilities for BPS projectors arise. In fact, the classical solutions to the deformed theory come in two types: Firstly, nonabelian solutions are continuously (in $\theta$ ) connected to their commutative counterparts (tensored with $\mathbb{1}_{\mathscr{H}}$ ) and represent smooth deformations of it. Secondly, abelian solutions become singular at $\theta \rightarrow 0$ and are genuinely noncommutative. In the BPS case, the simplest abelian projectors are of finite rank or co-rank in one copy of $\mathscr{H}$. Since novel features can be expected only in the abelian case, we focus on it from now on and choose $n=1$, i.e. the Moyal-deformed 
U(1) baby Skyrme model. Clearly, this theory permits abelian solutions only, since its commutative limit is free. However, it still contains an infinity of Grassmannian submodels corresponding to $\operatorname{Gr}(P)=\frac{\mathrm{U}(\mathscr{H})}{\mathrm{U}(\mathrm{im} P) \times \mathrm{U}(\operatorname{ker} P)}$ for some hermitian projector $P$, preferably of finite rank or co-rank $k$.

The Moyal deformation introduces the dimensionful parameter $\theta$ into the theory, which invalidates Derrick's argument: scaling of spatial coordinates now relates theories with different strengths of noncommutativity. Therefore, classical solutions at a fixed value of $\theta$ are safe against shrinking or spreading.

\section{Exact noncommutative baby Skyrmions}

The equations of section 2 carry over to the deformed abelian baby Skyrme model (with replacing $\mathbb{1}_{n}$ by $\mathbb{1}_{\mathscr{H}}$ ), since on a formal level its noncommutativity resembles the non-abelianness in the standard $\mathrm{U}(n)$ model. Hence, the failure of a standard noncommutative $\mathrm{U}(1)$ sigma-model BPS solution, $g=\mathbb{1}-2 P$ obeying (2.9), to also fulfil the baby Skyrme equation of motion, is again measured by (2.11). In our Moyal-deformed theory, this expression may vanish, and surprisingly does so if the projector is a function of the number operator $N=a^{\dagger} a$ only! In the star-product picture, this corresponds to functions only of the radial variable $r=\sqrt{z \bar{z}}$, and so they are called radial projectors. It is obvious that $F[P]$ in (2.11) vanishes for $P=P(r)$, but in the commutative theory only trivial projectors can be radial. In the Fock-space basis (3.3), radial projectors are simply diagonal.

Indeed, it is not hard to check explicitly that the BPS projector

$$
P^{(k)} \equiv \sum_{n=0}^{k-1}|n\rangle\langle n| \quad \text { obeys } \quad P_{\bar{z}}^{(k)} P_{z \bar{z}}^{(k)} P_{\bar{z}}^{(k)}=0=P_{z}^{(k)} P_{\bar{z} \bar{z}}^{(k)} P_{z}^{(k)}
$$

as well as $\left[P_{z \bar{z}}^{(k)}, P^{(k)}\right]=0$, in the sense of (3.4). Hence, $F\left[P^{(k)}\right]=0$, and the noncommutative baby Skyrme equation of motion is satisfied. In addition, due to the translation invariance of the model, the translates

$$
P^{(k, \alpha)} \equiv \mathrm{e}^{\alpha a^{\dagger}-\bar{\alpha} a} P^{(k)} \mathrm{e}^{-\alpha a^{\dagger}+\bar{\alpha} a} \quad \text { for } \quad \alpha \in \mathbb{C} \quad \text { and } \quad k \in \mathbb{N}
$$

also do the job. It is noteworthy that the role of $\operatorname{deg}[\phi]$ for the topological charge has been taken by the rank $k$ of the projector, which also defines a Grassmannian submanifold. Thus, for each value of $k$ we have found a $\mathbb{C}$-family of exact noncommutative $\mathrm{U}(1)$-valued baby Skyrmions, which are of course also solitons in the Grassmannian submodel. Most basic is the $k=1$ family

$$
P^{(1, \alpha)}=\mathrm{e}^{-\bar{\alpha} \alpha} \mathrm{e}^{\alpha a^{\dagger}}|0\rangle\left\langle 0\left|\mathrm{e}^{\bar{\alpha} a} \equiv\right| \alpha\right\rangle\langle\alpha| \quad \text { with } \quad a|\alpha\rangle=\alpha|\alpha\rangle
$$

which consists of the coherent-state projectors obtained by translating the ground-state projector $P^{(1)}=|0\rangle\langle 0|$. The corresponding function (under the Moyal-Weyl map) is just a Gaussian centered at $\alpha$ in the Moyal plane,

$$
P_{\star}^{(1, \alpha)}(z, \bar{z})=2 \mathrm{e}^{-|z-\alpha|^{2} / \theta},
$$

and the singular $\theta \rightarrow 0$ limit becomes apparent. 
Let us take a look at the energy of these configurations. The Grassmannian energy functional (2.6) reads

$$
E_{\mathrm{Gr}}[P]=16 \pi \theta \operatorname{Tr}_{\mathscr{H}}\left\{P_{z} P_{\bar{z}}+4 \kappa^{2}\left[P_{z}, P_{\bar{z}}\right]^{2}\right\}
$$

which for BPS projectors, due to $\left[P_{z}, P_{\bar{z}}\right]=P_{z \bar{z}}$, simplifies to

$$
E_{\mathrm{BPS}}[P]=16 \pi \theta \operatorname{Tr}_{\mathscr{H}}\left\{P_{z} P_{\bar{z}}+4 \kappa^{2} P_{z \bar{z}}^{2}\right\}=8 \pi \operatorname{Tr}_{\mathscr{H}}\left\{-\left[a^{\dagger}, P\right][a, P]+\frac{2 \kappa^{2}}{\theta}\left[a^{\dagger},[a, P]\right]^{2}\right\} .
$$

It is straightforward to evaluate this on the rank- $k$ diagonal projector of (4.1),

$$
E\left[P^{(k)}\right]=8 \pi \operatorname{Tr}_{\mathscr{H}}\left\{k|k\rangle\langle k|+\frac{2 \kappa^{2}}{\theta} k^{2}(|k\rangle\langle k|+| k+1\rangle\langle k+1|)\right\}=8 \pi\left(k+\frac{4 \kappa^{2}}{\theta} k^{2}\right) .
$$

Due to translation invariance, the same result holds for $P^{(k, \alpha)}$. The energy depends only on the dimensionless parameter $\kappa^{2} / \theta$. It exceeds the Bogomol'nyi bound of $8 \pi k$ by the contribution of the Skyrme term, whose $k^{2}$ dependence signals an instability of the higher-charge baby Skyrmions against decay into those of charge one. Interpreting $P^{(k)}$ as describing $k$ charge-one baby Skyrmions sitting on top of each other, they can lower their energy by passing to a configuration of near-infinite mutual separation, which is again a (near-exact) baby Skyrme solution. More general multi-center BPS solitons do not solve the baby Skyrme equation of motion (2.7), since they are not rotationally symmetric, and thus $F[P]$ does not vanish.

\section{Stability and interactions}

The baby Skyrmions of the full U(1) model are not stable due to the following argument: Consider a path in $\mathrm{U}(\mathscr{H})$ which connects a Grassmannian solution to the vacuum,

$$
g(s)=\mathrm{e}^{\mathrm{i}(\pi-s) P}=\mathbb{1}-\left(1+\mathrm{e}^{-\mathrm{i} s}\right) P \quad \text { with } \quad P^{\dagger}=P=P^{2} \quad \text { and } \quad s \in[0, \pi] .
$$

It interpolates between $g(0)=\mathbb{1}-2 P \in \operatorname{Gr}(P)$ and $g(\pi)=\mathbb{1}$. The energy

$$
\begin{aligned}
E(s) & =4 \pi \theta \operatorname{Tr}_{\mathscr{H}}\left\{\partial_{z} g^{\dagger} \partial_{\bar{z}} g+\kappa^{2}\left(\partial_{z} g^{\dagger} \partial_{\bar{z}} g-\partial_{\bar{z}} g^{\dagger} \partial_{z} g\right)^{2}\right\} \\
& =4 \pi \theta\left\{\left(1+\mathrm{e}^{\mathrm{i} s}\right)\left(1+\mathrm{e}^{-\mathrm{i} s}\right) \operatorname{Tr}_{\mathscr{H}}\left(P_{z} P_{\bar{z}}\right)+\kappa^{2}\left(1+\mathrm{e}^{\mathrm{i} s}\right)^{2}\left(1+\mathrm{e}^{-\mathrm{i} s}\right)^{2} \operatorname{Tr}_{\mathscr{H}}\left[P_{z}, P_{\bar{z}}\right]^{2}\right\} \\
& =8 \pi\left\{k \cos ^{2} \frac{s}{2}+\frac{4 \kappa^{2}}{\theta} k^{2} \cos ^{4} \frac{s}{2}\right\}
\end{aligned}
$$

along the path is decreasing monotonically to zero, which renders any soliton of the U(1) model unstable. This is not surprising, since the topological charge is well defined and conserved only inside the Grassmannian submanifolds.

To determine the long-range forces between two noncommutative baby Skyrmions, we compute the energy of a two-center BPS soliton, because for large separation this configuration approaches a superposition of two rank-one BPS solitons, which we have already found to be baby Skyrmions. In the two-center configuration

$$
P^{(\alpha, \beta)}=\frac{1}{1-|\sigma|^{2}}\{|\alpha\rangle\langle\alpha|+| \beta\rangle\langle\beta|-\sigma| \alpha\rangle\langle\beta|-\bar{\sigma}| \beta\rangle\langle\alpha|\} \quad \text { with } \quad \sigma=\langle\alpha \mid \beta\rangle
$$

the lumps are centered at positions $\alpha$ and $\beta$ in the complex Moyal plane, and the coherent states $|\alpha\rangle$ and $|\beta\rangle$ are normalized to one. This projector obeys the BPS condition (2.9) hence $\left[P_{z \bar{z}}^{(\alpha, \beta)}, P^{(\alpha, \beta)}\right]=$ 
0 but $F\left[P^{(\alpha, \beta)}\right] \neq 0$ unless $\alpha-\beta \rightarrow 0$ or $\infty$. Employing the defining relations $(a-\alpha)|\alpha\rangle=0$ and $(a-\beta)|\beta\rangle=0$ as well as $\sigma \bar{\sigma}=\mathrm{e}^{-|\alpha-\beta|^{2}}$, it is straightforward to compute

$$
\begin{aligned}
E\left[P^{(\alpha, \beta)}\right] & =8 \pi \operatorname{Tr}_{\mathscr{H}}\left\{-\left[a^{\dagger}, P^{(\alpha, \beta)}\right]\left[a, P^{(\alpha, \beta)}\right]+\frac{2 \kappa^{2}}{\theta}\left[a^{\dagger},\left[a, P^{(\alpha, \beta)}\right]\right]^{2}\right\} \\
& =8 \pi\left\{2+8 \frac{\kappa^{2}}{\theta}\left(1+\frac{1}{4} r^{4} \sinh ^{-2} \frac{r^{2}}{2}\right)\right\}
\end{aligned}
$$

where $r=|\alpha-\beta|$. This expression interpolates smoothly between

$$
\begin{aligned}
E\left[P^{(r=0)}\right] & =8 \pi\left(2+\frac{4 \kappa^{2}}{\theta} \cdot 4\right)=E\left[P^{(2)}\right] \\
E\left[P^{(r \rightarrow \infty)}\right] & =2 \cdot 8 \pi\left(1+\frac{4 \kappa^{2}}{\theta}\right)=2 \cdot E\left[P^{(1)}\right]
\end{aligned}
$$

which again underscores the decay channel $P^{(2)} \rightarrow P^{(1)}+P^{(1)}$. For large separation, the interaction potential is exponentially repulsive,

$$
V(r) \sim 64 \pi \frac{\kappa^{2}}{\theta} r^{4} \mathrm{e}^{-r^{2} / 2} \quad \text { for } \quad r \rightarrow \infty .
$$

Let us conclude by pointing out some open problems for future research. It would be interesting to find other exact abelian noncommutative baby Skyrmions or rule out this possibility; determine whether $P^{(1)}$ has minimal energy in the rank-one Grassmannian (i.e. is stable); and work out the scattering of two such lumps. Another promising task is to deform the full Skyrme model (on $\mathbb{R}^{1,3}$ ) and to construct noncommutative Skyrmions from noncommutative instantons [8].

\section{References}

[1] T. Ioannidou and O. Lechtenfeld, Phys. Lett. B 678 (2009) 508.

[2] G.S. Adkins, C.R. Nappi and E. Witten, Nucl. Phys. B 228 (1983) 552.

[3] T.H.R. Skyrme, Proc. Roy. Soc. Lon. 260 (1961) 127.

[4] A.H. MacDonald, Lecture Notes from NATO ASI "Quantum Transport in Semiconductor Submicron Structures", arXiv:cond-mat/9601144.

[5] J.A. Harvey, Komaba lectures on noncommutative solitons and D-branes, hep-th/0102076; A. Konechny and A.S. Schwarz, Phys. Rept. 360 (2002) 353 [arXiv:hep-th/0012145 \& 0107251]; M.R. Douglas and N.A. Nekrasov, Rev. Mod. Phys. 73 (2001) 977 [arXiv:hep-th/0106048]; R.J. Szabo, Phys. Rept. 378 (2003) 207 [arXiv:hep-th/0109162].

[6] O. Lechtenfeld and A.D. Popov, JHEP 0111 (2001) 040 [hep-th/0106213]; Phys. Lett. B 523 (2001) 178 [hep-th/0108118].

[7] O. Lechtenfeld, Noncommutative Solitons, in: noncommutative geometry and physics 2005, pp. 175-200, World Scientific 2007, arXiv:hep-th/0605034.

[8] M.F. Atiyah and N.S. Manton, Phys. Lett. B 222 (1989) 438;

T.A. Ioannidou, Nonlinearity 13 (2000) 1217 [arXiv:hep-th/0004174]. 